\title{
The description of the excitation energy sharing in nuclear fission within the Langevin approach
}

\author{
F.A. Ivanyuk $^{1,2, *}$ and $S$. Chiba ${ }^{2, * *}$ \\ ${ }^{1}$ Institute for Nuclear Research, 03028 Kiev, Ukraine \\ ${ }^{2}$ Tokyo Institute of Technology, 152-8550 Tokyo, Japan
}

\begin{abstract}
We apply the four-dimensional Langevin approach to the description of fission of ${ }^{235} \mathrm{U}$ by neutrons and calculate the dependence of the excitation energy of fission fragments on their mass number. For this we run the Langevin equations until the compound nucleus splits into two separated fragments. This is possible since the we used in this work two-center shell model shape parametrization that describes well both compact and separated shapes. The excitation energies of each fragment are calculated assuming that the temperatures of both fragments are the same. The deformation energy of the fragment immediately after scission is added to its excitation energy. The saw-tooth structure of the dependence neutron multiplicity on the fragment's mass number in reaction ${ }^{235} \mathrm{U}+\mathrm{n}$ at $E_{n}=5 \mathrm{Mev}$ is qualitatively reproduced.
\end{abstract}

\section{Introduction}

One of the most successful approaches to the description of fusion-fission reactions and the fission process is the approach based on the Langevin equations for the shape degrees of freedom. In this approach instead of co-ordinates of individual nucleons one introduces the parametrization of the shape of nuclear surface and solves the dynamical equations for the time evolution of deformation parameters and conjugated momenta. The internal state of the system in such calculations is assumed not to deviate much from the thermal equilibrium.

With the code developed at Tokyo Institute of Technology [1-5] during last few years we have managed to reproduce rather accurately the mass and total kinetic energy distribution of fission fragments. We have also found a very clear correlation between the neutron multiplicity and the deformation of fission fragment just before scission.

The existing descriptions of the sharing of excitation energy [4, 6-12] are based on very different assumptions and approximations that even contradict to each other. So, the reason of the saw-tooth structure at present is not clear. In this work we exploit the found correlation between the neutron multiplicity and the deformation of fission fragment in order to clarify the reason for the saw-tooth structure of neutron multiplicity and its dependence on the excitation energy.

\footnotetext{
*e-mail: ivanyuk@kinr.kiev.ua

**e-mail: chiba.satoshi@nr.titech.ac.jp
} 


\section{The model}

We describe the fission process within the Langevin approach [13], i.e., by solving the equations for the time evolution of the shape of nuclear surface of fssioning system. For the shape parametrization we use that os two-center shell model (TCSM) [14] with 4 deformation parameters $q_{\mu}=\left(z_{0} / R_{0}, \delta_{1}, \delta_{2}, \alpha\right)$. Here $z_{0} / R_{0}$ refers to the distance between the centers of left and right oscillator potentials with $R_{0}=1.2 \sqrt[3]{A}$ being the radius of spherical nucleus with mass number $A$. The parameters $\delta_{i}=3\left(a_{i}-b_{i}\right) /\left(2 a_{i}+b_{i}\right)$, where $\mathrm{i}=\{1,2\}$, describe the deformation of the right and left fragment tips. The fourth parameter $q_{4}$ is the mass asymmetry $\alpha=\left(V_{1}-V_{2}\right) /\left(V_{1}+V_{2}\right)$ where $V_{1}$ and $V_{2}$ are the volumes to the left and right from the point $z=0$. The fifth parameter of TCSM shape parametrization $\epsilon$, defined as the ratio of actual and oscillator potentials at $z=0, \epsilon \equiv E / E_{0}$, is kept constant, $\epsilon=0.35$, in all our calculations.

The first order differential equations (Langevin equations) for the time dependence of collective variables $q_{\mu}$ and the conjugated momenta $p_{\mathrm{mu}}$ are:

$$
\begin{aligned}
\frac{d q_{\mu}}{d t} & =\left(m^{-1}\right)_{\mu \nu} p_{\nu}, \\
\frac{d p_{\mu}}{d t} & =-\frac{\partial F(q, T)}{\partial q_{\mu}}-\frac{1}{2} \frac{\partial m_{\nu \sigma}^{-1}}{\partial q_{\mu}} p_{\nu} p_{\sigma}-\gamma_{\mu \nu} m_{\nu \sigma}^{-1} p_{\sigma}+g_{\mu \nu} R_{v}(t),
\end{aligned}
$$

where the sums over the repeated indices are assumed. In Eqs. (1) $F(q, T)$ is the temperature dependent free energy of the system, and $\gamma_{\mu \nu}$ and $(m)_{\mu \nu}^{-1}$ are the friction and inverse of mass tensors and $g_{\mu \nu} R_{v}$ is the random force.

The free energy $F(q, T)$ is calculated as the sum of liquid drop deformation energy and the shell correction $\delta F(q, T)$. The damping of shell correction $\delta F(q, T)$ with the excitation energy is described in detail in [15]. The single particle energies are calculated with the deformed Woods-Saxon potential [16-18] fitted to the aforementioned TCSM shape parameterizations. The collective inertia tensor $m_{\mu \nu}$ is calculated within the Werner-Wheeler approximation [19] and for the friction tensor $\gamma_{\mu \nu}$ we used in present work the wall-and-window formula [20,21].

The random force $g_{i j} R_{j}(t)$ is the product of white noise $R_{j}(t)$ and the temperature dependent strength factors $g_{i j}$. The factors $g_{i j}$ are related to the temperature and friction tensor via the modified Einstein relation [22],

$$
g_{i k} g_{k j}=T^{*} \gamma_{i j}, \quad \text { with } T^{*}=\frac{\hbar \varpi}{2} \operatorname{coth} \frac{\hbar \varpi}{2 T},
$$

where $T^{*}$ is the effective temperature. The parameter $\varpi$ is the local frequency of collective motion [22]. The minimum of $T^{*}$ is given by $\hbar \varpi / 2$, which corresponds to the zero point energy of oscillators forming the heat bath. Based on the pioneering works [23, 24], we estimated the zero point energy as $1 \mathrm{MeV}$, which lies in the middle of the corresponding quantities for various modes 0.45 to $2.23 \mathrm{MeV}$ estimated in Ref. [24]. The temperature $T$ in this context is related to the initial excitation energy $E_{x}$ and the internal energy $E_{\text {int }}$ by,

$$
E_{\mathrm{int}}=E^{*}-\frac{1}{2}\left(m^{-1}\right)_{i j} p_{i} p_{j}-V(q, T=0)=a T^{2},
$$

where $a$ is the level density parameter.

More details are specified in our earlier publications [1-3].

Initially, the momenta $p_{i}$ were set to zero, and calculations are started from the ground state deformation. Such calculations are continued until the trajectories reach the "scission point", which was defined as the point in deformation space with a certain value of neck radius. 


\section{Numerical results}

A single event of Langevin calculation typically begins from the ground state deformation. If it fails to achieve scission configuration after $10,000 \mathrm{fm} / \mathrm{c}$, the calculation is terminated. Usually 500,000 events are done per nuclei but we occasionally increase the number of events if it was a critical calculation. Typically, the trajectories stay for a lot of time inside that
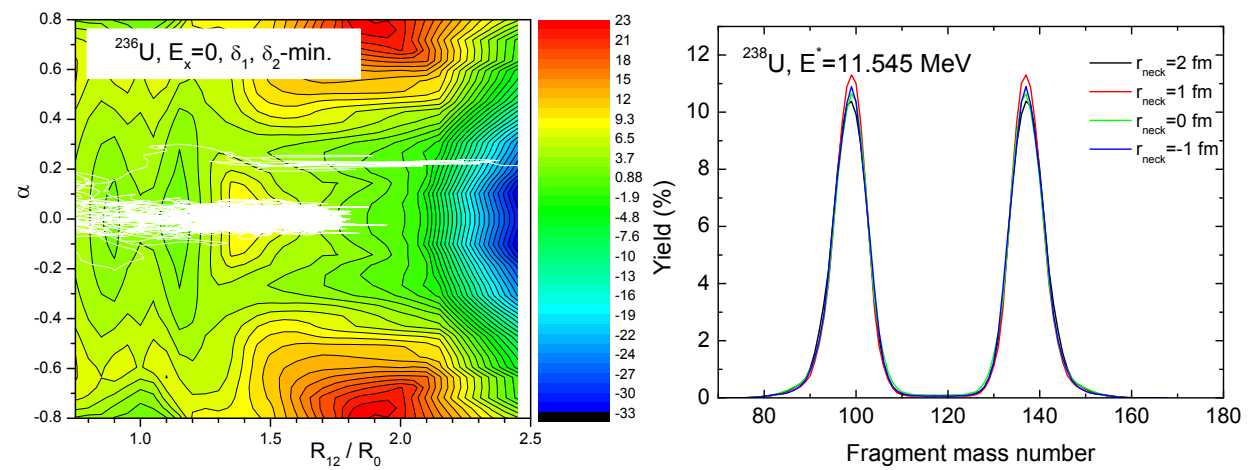

Figure 1. Left: the potential energy of ${ }^{236}$ in elongation $R_{12}$ and mass asymmetry $\alpha$ coordinates, minimized with respect to $\delta_{1}$ and $\delta_{2}$ deformation parameters. White line is an example of trajectory in deformation space. Right: the mass distribution of fission fragments calculated at different critical value of the neck parameter, $r_{\text {neck }}=2,10$ and $-1 \mathrm{fm}$.

saddle, some of them go over the saddle and reach the scission point, see Fig. 1a. In our previous calculations we defined the scission point as a deformation at which the neck radius turns into zero. However there are some indications that the fissioning nucleus breaks apart at rather thick neck. This was shown a long ago within the liquid drop model [25]. The calculations within Time-Dependent Hartree-Fock approach are also stopped at the finite value of the neck of the order of 1-2 fm.

In Fig. 1b we compare the mass distribution of fission fragment of ${ }^{236} U$ at the excitation energy $E^{*}=11.545 \mathrm{MeV}$. As one can see, the mass distributions are rather stable with respect to variation of $r_{\text {neck }}$ within a reasonable limits, $2 \mathrm{fm} \leq r_{\text {neck }} \leq-1 \mathrm{fm}$. The negative neck radius refer here to the separated fragments, see Fig. 2.

In our previous publication [4] we stop the calculations when the neck radius turns into zero. Then we fitted this just before scission shape by the shape of two separated fragments and calculated the deformation and excitation energies of each fragments separately. Since the scission point is not well defined, in present work we avoid the fitting of just before scission shape and continue Langevin calculations until the compact shape would split into two fragments. This is possible since the TCSM shape parametrization describes well both compact and separated shapes. Practically, we run calculations until the neck parameter became negative, $r_{\text {neck }}=-1 \mathrm{fm}$. Then for each fragment we calculated the deformation and excitation energies. The deformation energy was calculated by the macroscopic-microscopic method, $E_{\text {def }}(q)=E_{\mathrm{LDM}}(q)+\delta E_{\text {shell }}(q)+\delta E_{\text {pair }}(q)$, the same as for the compact configuration, and for the thermal excitation energy we used the BCS-approximation,

$$
E^{*}(T)=E_{\mathrm{BCS}}\left(q^{(\mathrm{ias})}, T\right)-E_{\mathrm{BCS}}\left(q^{\text {(ias) }}, T=0\right)
$$

Here $q^{\text {(ias) }}$ is the set of deformation parameters "immediately after scission" (at $r_{\text {neck }}=$ $-1 \mathrm{fm})$. The temperature of light and heavy fragments immediately after scission was assumed to be the same and was found from the requirement that the sum of excitation energies 


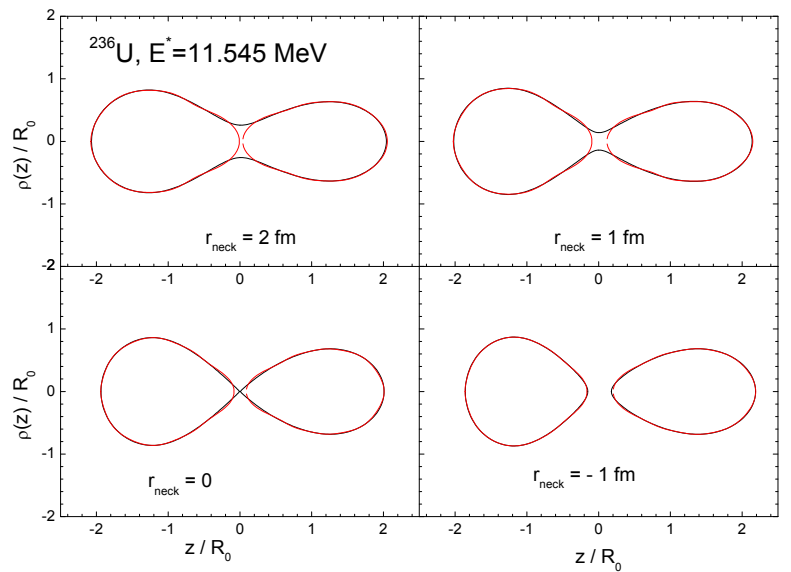

Figure 2. The shape of fissioning ${ }^{236} \mathrm{U}$ at critical neck radius $r_{\text {neck }}=2,1,0$ and $-1 \mathrm{fm}$.

of light and heavy fragments is equal to the total excitation energy $E_{\text {int }}$, see Eq. (3), resulting from the Langevin calculations,

$$
E_{L}^{*}(T)+E_{R}^{*}(T)=E_{\text {int }}
$$

In Fig. 3a we plot separately the ground state energy $E_{\mathrm{gs}}$, the deformation energy $E_{\mathrm{def}}^{(\mathrm{ias})}$ and the excitation energy $E^{*}$ as function of fragment mass number. One can see that the main
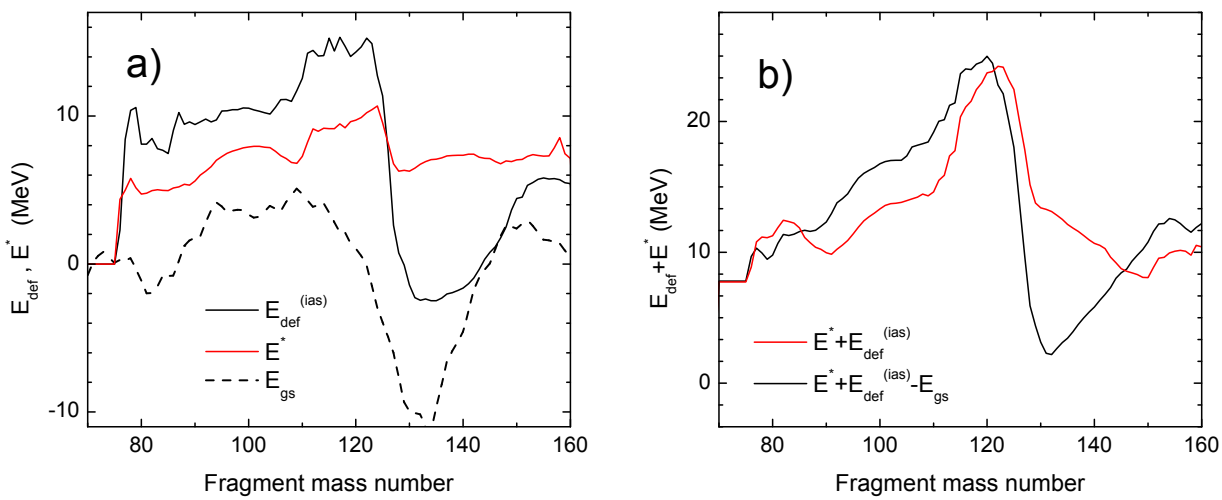

Figure 3. a) The average value of the ground state energy $E_{\mathrm{gs}}$ (dash), deformation $E_{\text {def }}^{\text {(ias) }}$ (black) and excitation $E^{*}$ (red) energies of fission fragments after the neck rupture as function of the fragment mass number; b) the sum $E^{*}+E_{\mathrm{def}}^{\text {(ias) }}$ (black) and the total excitation energy $E^{*}+E_{\mathrm{def}}^{(\mathrm{ias})}-E_{\mathrm{gs}}$ as function of the fragment mass number.

contribution of the saw-tooth structure of excitation energy comes mainly from the deformation energy of fission fragment. This result is not new. It was noticed by Wilkins et al [26] already in 1976 that the dependence of quadrupole moment os fission fragment on its mass number is very similar to that of neutron multiplicity. The sharing of excitation energy seems not so important. Even if one would share the excitation equally between the fragments, or share it proportionally to the mass of fragment, the saw-tooth structure would remain due to $E_{\text {def }}^{\text {(ias) }}$. 
The sum $E^{*}+E_{\mathrm{def}}^{\text {(ias) }}$ surprisingly well reproduces the structure of neutron multiplicity, see the left part of Fig. 4. In the right part of Fig. 4 we compare the experimental values of average neutron multiplicity $\bar{v}$ from [27], with that derived from the excitation energy $E^{*}+E_{\mathrm{def}}^{(\mathrm{ias})}$. For $\bar{v}$ we have calculated the decay of excited fission fragments by Hauser-Feshbach theory as implemented in Ref. [28]. The transmission coefficients for neutron and proton emission (if any) were calculated by the spherical optical model by using optical potential parameters suggested by Koning and Delaroche [29]. The level densities of the residual nuclei were calculated by Gilbert-Cameron composite formula with parameters given in Ref. [30]. At low excitation energies, the discrete level data were taken from the evaluated nuclear structure data stored in RIPL-3 [31] (updated in 2012). For $\gamma$ emission, we have included E1, E2 transitions, and M1 spin-flip and scissors modes. We have fixed the spin and parity of the decaying fragment in this work for simplicity. We tried calculation with $J^{\pi}=0^{+}$for even and $\frac{1}{2}^{+}$for odd nuclei, but the results do not change appreciably if we increase spin values by $8 \hbar$. We recognize that we have to assign distributions of spin and parity as performed in Ref. [28] for final calculation, but a "fixed" spin-parity treatment should be enough as an initial investigation.
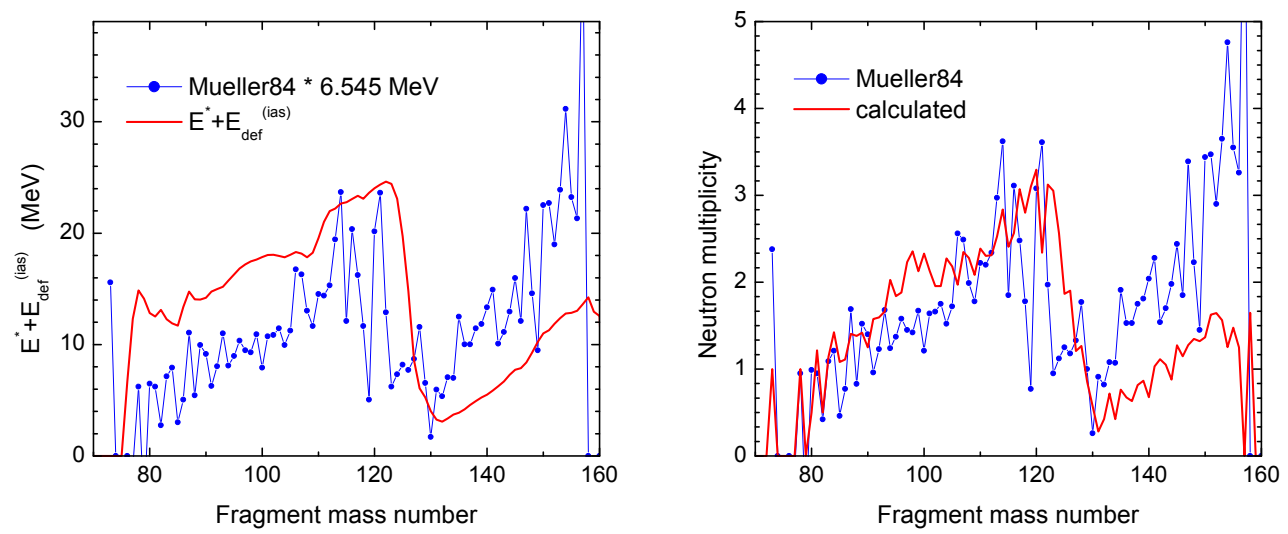

Figure 4. Left: the sum of the excitation and the deformation energy (red) in comparison with the experimental value of $\bar{v}$ multiplied by the neutron separation energy. Right: the comparison of the calculated and experimental values of $\bar{v}$.

As one can see from the left part of Fig. 4, the saw-tooth structure of $\bar{v}$ is well reproduced. The difference between the measured and calculated $\bar{v}$ for light fission fragments is within the experimental uncertainties. For the heavy fragments the calculated $\bar{v}$ is somewhat smaller.

Here we should note that $E^{*}+E_{\mathrm{def}}^{(\mathrm{ias})}$ is only part of excitation energy. It is believed that the prompt neutrons are emitted from completely accelerated fragments, thus, from the fragments at their ground state deformation. The total excitation energy available for the neutron emission is then $E^{*}+E_{\mathrm{def}}^{(\mathrm{ias})}$ minus the ground state energy $E_{\mathrm{gs}}, E_{\mathrm{tot}}^{*}=E^{*}+E_{\mathrm{def}}^{(\mathrm{ias})}-E_{\mathrm{gs}}$. The subtraction of $E_{\mathrm{gs}}$ destroys significantly the similarity of $\bar{v}$ and $E_{\mathrm{tot}}^{*}$. The minimum of $E^{*}$ moves from $A_{F} \approx 132$ to $A_{F} \approx 150$. Consequently, the agreement between experimental and calculated values of $\bar{v}$ became worth, see Fig. 5.

We do not see what might be wrong in our approach. Once we got the shape of separated fragments from output of Langevin approach, the calculating of deformation and excitation energies is done exactly, without any approximation, no fitted parameters like levels density parameter or damping of the shell correction. All the important properties of fissioning system, the shell effects, the pairing effects, the dependence of both on the excitation energy, are 

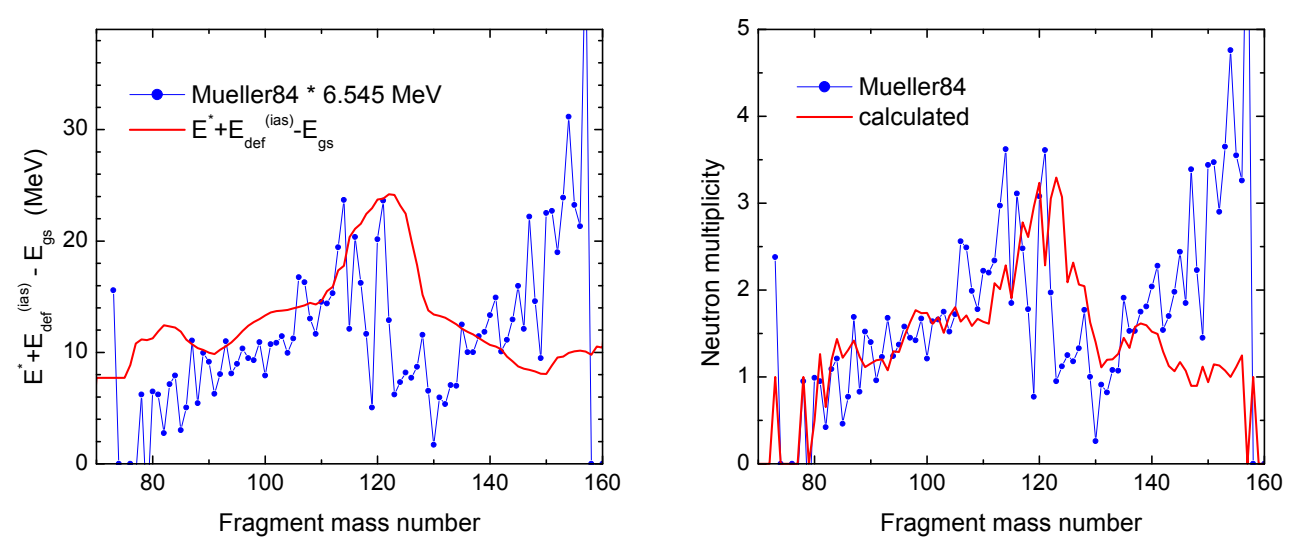

Figure 5. Left: the sum of the excitation $E^{*}$ and the extra deformation energy $E_{\mathrm{def}}-E_{\mathrm{gs}}(\mathrm{red})$ in comparison with the experimental value of $\bar{v}$ multiplied by the neutron separation energy. Right: the comparison of the calculated and experimental values of $\bar{v}$.

taken into account. In our opinion the similar problem with the subtraction of ground state energy should have other authors who explain the saw-tooth structure of $\bar{v}$.

In this work we calculate $E_{\text {def }}^{\text {(ias) }}$ for each fragment with respect to the macroscopic energy at the spherical shape. After the neck rupture the fragments relax to the spherical shapes (because they are still excited) within the very short relaxation time, $\tau \approx 5 \times 10^{-22} \mathrm{sec}$. Hence, what we calculate is the neutron emission in the very early stage of acceleration. Since we explain well the data one can infer that the neutron emission takes place shortly after scission.

The fact that the prompt neutrons are registered at the same direction as the fission fragment does not say much at what moment the neutron are actually emitted. Some part of neutrons may be emitted at the acceleration stage, before they relaxed to their ground state shape. One should keep in mind also the possibility of scission neutrons [32] which are emitted during the neck rupture, thus, from highly deformed fragments. The number of scission neutrons is not so small, it can reach one half of all prompt neutrons [33].

\section{Summary}

We used the output of the 4D Langevin approach to calculate the excitation energies of fission fragments in reaction ${ }^{235} \mathrm{U}+\mathrm{n}$ at neutron energies $E_{n}=5 \mathrm{MeV}$. The Langevin code was run until the compact system would split into tao fragments.

The temperature of fission fragments was assumed to be the same and was found from the requirement that the sum of excitation energy of fission fragments immediately after scission is equal to the excitation energy of fissioning nucleus. We have found that the dependence of excitation plus deformation energy on the fragment mass number surprisingly well reproduces the saw-tooth structure of average neutron multiplicity $\bar{v}$. This can be considered as a hint that a considerably part of prompt neutrons is emitted not from the fully accelerated fragments but on the acceleration stage or during the neck rupture. The subtraction of ground state deformation energy from fragments excitation energy makes the agreement with experimental data much worth. 
This study comprises the results of "Research and development of an innovative transmutation system of LLFP by fast reactors" entrusted to the Tokyo Institute of Technology by the Ministry of Education, Culture, Sports, Science and Technology of Japan (MEXT) and KAKENHI Grant Number 18K03642 from Japan Society for the Promotion of Science (JSPS). One of us (F.I.) was supported in part by the project "The investigation of statistical properties of collective states and nuclear fission of rear-earth and actinide nuclei" of the National Academy of Sciences of Ukraine.

\section{References}

[1] F.A. Ivanyuk, S. Chiba, and Y. Aritomo, Jour. Nucl. Sci. Tech. 53, 737 (2016)

[2] M.D. Usang, F.A. Ivanyuk, C. Ishisuka, and S. Chiba, Phys. Rev. C 94, 044602 (2016)

[3] C. Ishizuka, M.D. Usang, F.A. Ivanyuk et al., Phys. Rev. C 96, 064616 (2017)

[4] F.A. Ivanyuk, C. Ishizuka, M.D. Usang, and S. Chiba, EPJ Web of Conferences 169, 00005 (2018)

[5] M.D. Usang, F.A. Ivanyuk, C. Ishisuka, and S. Chiba, Scientific Reports 9, 1525 (2019)

[6] K.-H. Schmidt and B. Jurado, Phys. Rev. Lett. 104, 212501 (2010)

[7] K.-H. Schmidt and B. Jurado, Phys. Rev. C 83, 061601(R) (2011)

[8] C. Morariu, A. Tudora, F.-J. Hambsch, S. Oberstedt, C. Manailescu, J. Phys. G. Nucl. Part. Phys. 39, 055103 (2012)

[9] B. Becker, P. Talou, T. Kawano, Y. Danon, and I. Stetcu, Phys. Rev. C 87, 014617 (2013)

[10] A. Tudora, F.-J. Hambsch, I. Visana, G. Giubega, Nucl. Phys. A 940, 242 (2015)

[11] M. Albertsson, B.G. Carlsson, T. Dossing, P. Moller, J. Randrup and S. Aberg, preprint nucl-th/1811.02283 (2018)

[12] A. Bulgac, S. Jin, K.J. Roche et al., Phys. Rev. C 100, 034615 (2019)

[13] Y. Abe, S. Ayik, P.-G. Reinhard and E. Suraud, Phys. Rep. 275, 49 (1996)

[14] J. Maruhn and W. Greiner, Z. Phys. 251, 431 (1972)

[15] F.A. Ivanyuk, C. Ishizuka, M.D. Usang and S. Chiba, Phys. Rev. C 97, 054331 (2018)

[16] V.V. Pashkevich, Nucl. Phys. A 169, 275 (1971)

[17] V.V. Pashkevich, Nucl. Phys. A 477, 1 (1988)

[18] V.V. Pashkevich, A.Ya. Rusanov, Nucl. Phys. A 810, 77 (2008)

[19] K.T.R. Davies, A.J. Sierk, J.R. Nix, Phys. Rev. C 13, 2385 (1976)

[20] J. Blocki, Y. Boneh, J.R. Nix et al, Ann. Phys. 113, 330 (1978)

[21] A.J. Sierk and J.R. Nix, Phys. Rev. C 21, 982 (1980)

[22] H. Hofmann, D. Kiderlen, Int. Jour. Mod. Phys. E 7, 243 (1998)

[23] N. Bohr and A.J. Wheeler, Phys. Rev. 56, 426 (1939)

[24] D.L. Hill and A.J. Wheeler, Phys. Rev. 89, 1102 (1952)

[25] V.M. Strutinsky, N. Ya. Lyashchenko, N.A. Popov, Nucl. Phys. 46, 659 (1963)

[26] B.D. Wilkins, E.P. Steinberg, R.R. Chasman, Phys. Rev. C 14, 1832 (1976)

[27] R. Müller, A.A. Naqvi, F. Käppeler, and F. Dickmann, Phys. Rev. C 29, 885 (1984)

[28] S. Okumura et al., J. Nucl. Sci. Tech., 55, 1009 (2018)

[29] A.J. Koning and J.P. Delaroche, Nucl. Phys. A 713, 231 (2003)

[30] T. Kawano, S. Chiba and H. Koura, Nucl. Sci. Tech., 43, 1 (2016)

[31] R. Capote et al., Nucl. Data Sheets 110, 3107 (2009)

[32] N. Carjan and M. Rizea, Phys. Rev. C 99, 034613 (2019)

[33] N. Carjan, Contribution to Theory-5 Workshop 